\title{
ACUMULACIÓN DE PLOHO EN ESPECIAS: POTENCIAL PARA PROCESOS DE FITORREMEDIACIÓN DE SUELOS URBANOS CONTAMINADOS EN ARGENTINA
}

\author{
LEAD ACCUMULATION IN SPICES: POTENTIAL \\ FOR PHYTOREMEDIATION OF URBAN \\ CONTAMINATED SOILS IN ARGENTINA
}

Mayra Agustina Rios Medrano mriosmedrano@uade.edu.ar

Estudiante avanzada de la Licenciatura en Biotecnología en la Universidad Argentina de la Empresa.

Judith Elizabeth Lacava julacava@uade.edu.ar Licenciada en Gestión Ambiental por la Universidad Argentina de la Empresa.

\section{Ignacio Curotto icurotto@uade.edu.ar}

Estudiante de la Licenciatura en Biotecnología en la Universidad Argentina de la Empresa.

\section{Leandro Fuks lefuks@uade.edu.ar}

Estudiante avanzado de Ingeniería en Alimentos en la Universidad Argentina de la Empresa.

\section{Romina Mariel Gargarello rgargarello@uade.edu.ar}

Doctora en Ciencias Básicas y Aplicadas por la Universidad Nacional de Quilmes. Investigadora del Instituto de Tecnología, Universidad Argentina de la Empresa. 


\section{RESUMO}

En Argentina no existe información unificada y fácilmente accesible en relación con el potencial de acumulación de metales pesados en especies vegetales ${ }^{1}$. El avance en estudios de acumulación de los principales contaminantes metálicos conformaría una herramienta importante para la gestión y recuperación de suelos contaminados, a partir del diseño de procesos de fitorremediación. Particularmente, el plomo es un contaminante común en las regiones pobladas del Gran Buenos Aires (AMBA), dada la actividad industrial predominante en esas zonas y que han generado un gran pasivo ambiental. En este trabajo se presentan resultados de acumulación de plomo en condimentos, a escala laboratorio, de cebolla de verdeo y orégano. Las especies elegidas para este estudio representan condimentos de amplio uso en la cocina argentina y de las primeras opciones disponibles para comenzar una huerta doméstica. Los resultados indican una alta capacidad de acumulación, que permitió remover entre el 80 y $90 \%$ del plomo presente en suelos o cuerpos de agua. Estos resultados preliminares dan indicios de la factibilidad de utilizar estas especies en procesos de fitorremediación².

Palavras-chave: Metales pesados. Plomo. Condimentos. Fitorremediación.

\section{ABSTRACT}

In Argentina, there is no unified and easily accessible information regarding the potential for accumulation of heavy metals in plant species. Goals in accumulation studies of the main metallic pollutants would be an important tool for the management and recovery of contaminated soils, based on the design of phytoremediation processes. Particularly, lead is a common pollutant in the most populated regions of Greater Buenos Aires (AMBA), as a consecuence of industrial activity that have generated a large environmental liability. In this paper results of laboratory scale lead accumulation experiments in spices such as green onion and oregano are presented. The vegetables chosen for this study represent spices widely used in argentinian cuisine and the first available options to start a domestic garden. The results indicate a high accumulation capacity, which allowed to remove between 80 and $90 \%$ of the lead present in soils or water. These preliminary results are an advance of the feasibility of using these species in phytoremediation processes.

Keywords: Heavy metals. Lead. Spices. Phytoremediation.

\footnotetext{
${ }^{1}$ Al momento de esta presentación.

${ }^{2}$ Investigación realizada en el marco del proyecto de investigación "Acumulación de Metales de Riesgo Toxicológico en Vegetales Consumibles", financiado por la Universidad Argentina de la Empresa.
} 


\section{INTRODUÇÃo}

En los últimos años, el crecimiento de actividades agrícolas periurbanas y domésticas (tales como huertas agroecológicas, orgánicas, urbanas o escolares) representan una oportunidad en la búsqueda de la independencia alimenticia, el desarrollo local, la generación de puestos de trabajo y el acceso a alimentos de calidad. Organizaciones como FAO, indican que, a nivel mundial, unos 815 millones de personas sufren inseguridad alimentaria y aproximadamente 2000 millones no disponen de alimentos suficientemente nutritivos; mientras que el $95 \%$ de los alimentos provienen del suelo y que el $33 \%$ de los suelos del planeta están degradados (FAO, 2018). Asimismo, ante el crecimiento poblacional y la dificultad de acceso a alimentos, los programas de promoción de huertas urbanas van tomando presencia, como herramienta de aseguramiento nutricional y desarrollo local (FAO, 2009; JANINE SCHONWALD, 2015; INTA, 2018). En este marco, existen experiencias registradas en diversos países, como Kenya (OWOUR, 2008), Brasil (MADALENO, 2000), México (DIELEMAN, 2017) y Tanzania (ZELLA, 2018), entre otros.

En Argentina una de las zonas con mayor densidad poblacional es la denominada AMBA- Área Metropolitana de Buenos Aires conformada por la Ciudad Autónoma de Buenos Aires (capital del país) y 40 municipios aledaños. Esta región concentra aproximadamente el 32\% de la población total de Argentina, según el último censo nacional (IGN, 2010).

Desde el año 1990, el Instituto Nacional de Tecnología Agropecuaria- INTA- Ileva adelante un programa de fortalecimiento de prácticas productivas para el autoabastecimiento, denominado ProHuerta. Hasta el año 2011, la coordinación nacional ProHuerta llevaba registradas 74.968 huertas en la región del AMBA, entre huertas urbanas, domésticas y escolares (INTA, 2011).

En contraposición, existen riesgos relacionados a la calidad de los alimentos producidos, vinculados con la calidad del suelo y el agua de riego. En las áreas urbanas suele encontrarse que los horizontes superficiales de los suelos están contaminados con metales, metales pesados y sustancias orgánicas, de fuentes difusas, como tránsito vehicular, o fuentes puntuales como emisiones industriales (GIUFFRÉ, 2014).

Los metales pesados y sus compuestos no son biodegradables, se acumulan y perpetúan en el medio ambiente, en los organismos y en las cadenas tróficas, impactando negativamente en la salud humana y en los ecosistemas. La contaminación antrópica con metales pesados representa un riesgo muy importante para las comunidades que conviven en zonas cercanas a actividades industriales, como ocurre en gran parte del AMBA, Argentina.

Existe evidencia de la capacidad acumuladora de varias especies vegetales presentes en la horticultura urbana como solanáceas, cucubitáceas, brásicas (XUEZHU YE, 2014) y vegetales de hoja 
verde como espinaca (YING CHEN, 2014) y rúcula (XIAOYAN TANG, 2018). Los metales ensayados corresponden a actividades industriales urbanas, como arsénico, plomo, cromo, cobre y níquel, entre otros (FERNANDO GUERRA, 2012). Los valores encontrados en algunos casos superan los límites establecidos por los respectivos códigos alimentarios, haciendo inviable su consumo. Sin embargo, la gran capacidad acumuladora de metales de estas especies haría posible incluirlas como parte de una planificación de manejo del suelo previo al uso para agricultura doméstica, basada en la fitorremediación.

Para la región de estudio AMBA, existen algunos estudios previos vinculados a la calidad de suelo (FAGGI, 2006; GIUFFRÉ, 2013) que reportan concentraciones de metales considerablemente preocupantes para la agricultura doméstica, pero no incursionan con intensidad en la acumulación resultante de metales en vegetales a partir del cultivo en suelo, sustrato o sistemas sin sustrato. Asimismo, una problemática de contaminación antrópica incipiente está relacionada con la presencia de altos niveles de plomo, pasivo ambiental resiliente de la industria automotriz y petroquímica, presente en la región (GAISO, 2014). La calidad de suelos para cultivo, en el caso de la instalación de huertas domésticas, es examinada en el caso que sean instrumentadas por organismos oficiales. Sin embargo, ante la creciente demanda y percepción de acceso a alimentos más saludables, no todas las huertas se inscriben en programas oficiales, representando un riesgo para la salud el consumo de hortalizas cultivadas en suelos de baja calidad.

La propuesta de este trabajo es vincular la actividad hortícola urbana con procesos de recuperación de suelos, detectando especies acumuladoras de metales, potencialmente utilizadas para etapas de remediación previas a la siembra de especies consumibles, basados en las especies más sembradas por los habitantes de la región y consumidas en la mesa argentina, como son las especias.

\section{METODOLOGIA}

Para la realización de este trabajo se utilizaron como modelos vegetales de experimentación, cebolla de verdeo (Allium fistulosum) y orégano (Origanum vulgare) en dos sistemas de cultivo: con sustrato sólido y sin sustrato. Los sistemas fueron incubados en un cuarto de cultivo vegetal a $25^{\circ} \mathrm{C} \pm 2$ y fotoperiodo controlado, $11 \mathrm{hs}$. luz- $13 \mathrm{hs}$. oscuridad. Los plantines utilizados para este trabajo fueron adquiridos en un vivero especializado dedicado a la comercialización para el sector agropecuario de la provincia de Buenos Aires, a los 30 días de siembra.

\subsection{CULTIVO SIN SUSTRATO}

Se ubicaron 40 plantines en soportes inertes (plástico) y se expusieron 20 unidades a $5 \mathrm{~mL}$ de una solución de contacto de $100 \mathrm{mg} / \mathrm{L}$ de plomo (Pbll; a partir de nitrato de plomo), pH 4.5, durante 15 días, a 
volumen constante. Otros 20 plantines fueron destinados para control, utilizando agua de grifo sin cloro, pH 5. Cada sistema se realizó por duplicado.

\subsection{CULTIVO EN SUSTRATO.}

Se ubicaron 40 plantines en macetas plásticas con sustrato comercial, proveniente de compost orgánico. Para cada maceta, se utilizaron 50gr de un preparado de sustrato- perlita en relación 1:0,2. Todos los sistemas se regaron durante 15 días. 20 unidades fueron regadas con solución $100 \mathrm{mg} / \mathrm{L}$ de plomo (Pbll; a partir de nitrato de plomo), pH 4.5. El riego se diseñó de manera que a los 7 días de comenzado el experimento, el sustrato cuente con $300-400$ gPb/g sustrato, según lo encontrado en condiciones de contaminación antrópica por (Faggi, 2006). Otras 20 unidades fueron destinadas para control, utilizando agua de grifo sin cloro, pH 5, para el riego. Cada sistema se realizó por duplicado.

\subsection{PARÁMETROS FISICOQUÍMICOS.}

En todos los casos se midió pH utilizando electrodo combinado.

Para el sustrato, se midió pH actual, utilizando una relación sustrato: agua de 1:1, homogeneizando y dejando reposar durante 30 minutos.

La materia orgánica fue cuantificada por gravimetría y calcinación a $550{ }^{\circ} \mathrm{C}$ según el método de Storer (STORER, 1984).

La humedad del sustrato fue cuantificada por gravimetría con incubación a $150{ }^{\circ} \mathrm{C}$.

\subsection{COSECHA Y MEDICIÓN DEL CONTENIDO DE PLOMO}

Finalizado el tiempo de contacto o riego, se realizó la cosecha y separación manual de tallo y raíz de cada sistema. Una vez separados los tejidos, tallos y raíces de cada sistema se secaron en estufa a $60^{\circ} \mathrm{C} \mathrm{y}$ se molieron en mortero. El plomo acumulado en los vegetales y el remanente en sustrato fue recuperado por extracción ácida (método EPA3050B). La cuantificación de plomo fue realizada por Espectroscopía de Absorción Atómica de Llama. 


\subsection{FACTOR DE TRANSFERENCIA.}

El factor de transferencia, TF, fue calculado según lo propuesto por Malik (Malik, 2014), utilizando la siguiente ecuación:

\section{Ecuación 1:}

$$
T F=\frac{C_{P b^{\text {suelo }}}}{C_{P b^{\text {planta }}}}
$$

Donde $\mathrm{C}=$ concentración de metal en $\mathrm{mg} / \mathrm{L}$

\section{RESULtados e ANÁLISE}

Los resultados muestran acumulación de plomo en cantidades considerables. En la Figura 1, se muestra la acumulación de plomo en raíces y tallos para orégano y cebolla de verdeo crecidos en sustrato sólido.

Mientras que el Plomo se acumuló en raíces y tallos para la cebolla de verdeo, no fue detectado en raíces de orégano. Para estos ensayos, los balances de masa calculados a partir de la cantidad de Plomo agregado, remanente en sustrato y acumulado en los vegetales, equivalen a $87,5 \%$ de remoción de Plomo para cebolla de verdeo y $86,4 \%$ para orégano.

El factor de transferencia calculado para cada especie arrojó valores similares: 0,40 para verdeo y 0,42 para orégano. 
Figura 1 - Acumulación de Plomo en orégano y cebolla de verdeo cultivados con sustrato. La desviación standard para cada serie no es significativa $(<0,03)$. La cantidad de plomo acumulado en raíz de orégano no fue significativa $(<0,0001)$. Los resultados se expresan en $\mathrm{mg} / \mathrm{Kg}$ de biomasa seca

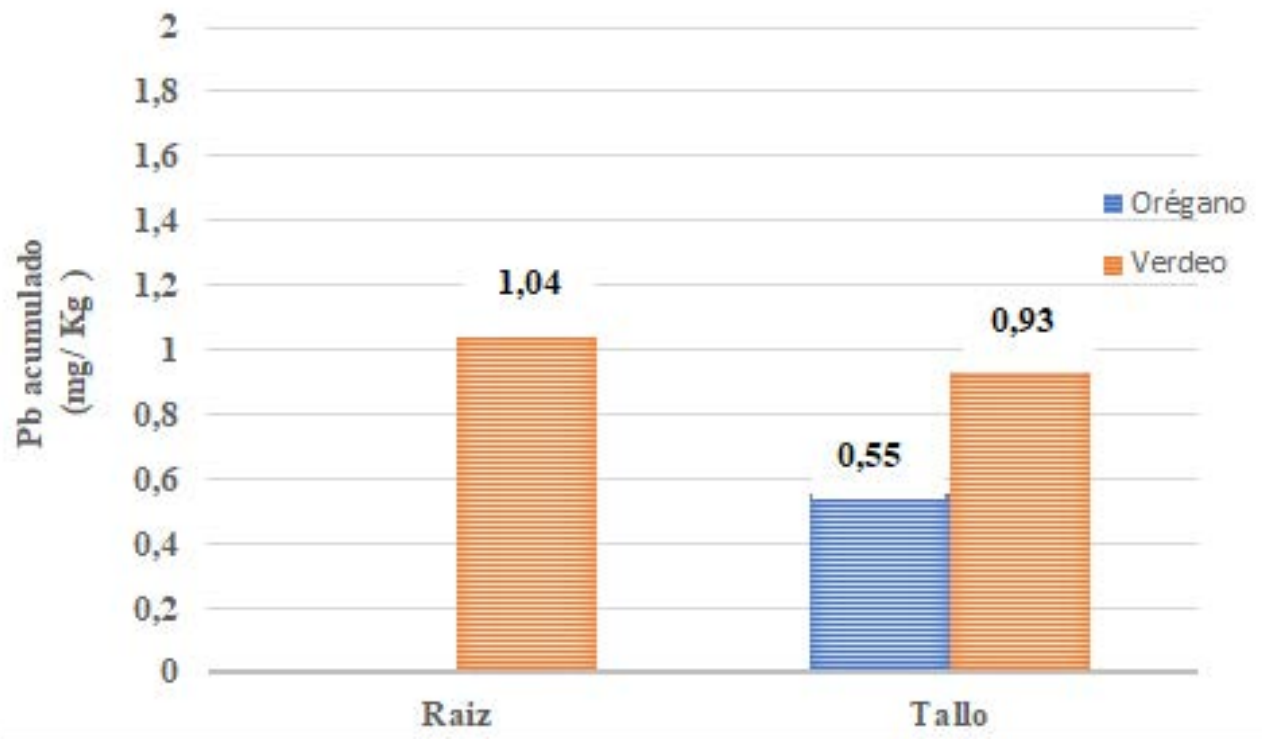

Fuente: Elaboración de la autora

Las experiencias sin sustrato fueron diseñadas con propósito de simular casos de cultivo en acuaponia. Los resultados se presentan en la Figura 2.

En estas condiciones se observa un importante aumento en la cantidad de Plomo acumulada en raíces y tallos de ambas especies en estudio, siendo la cebolla de verdeo el condimento que mayor capacidad de remoción presenta.

El inconveniente encontrado con este ensayo se vincula a precipitados encontrados hacia el día 13 de la experiencia, probablemente debido a la interacción del plomo con detritos celulares y exudados radiculares. Esto imposibilitó realizar un balance de masa y calculo del factor de transferencia confiables, dado que la concentración de metal remanente en solución al día 15 fue no detectable. 
Figura 2 - Acumulación de Plomo en orégano y cebolla de verdeo cultivados sin sustrato. La desviación standard para cada serie no es significativa $(<0,02)$. Los resultados se expresan en $\mathrm{mg} / \mathrm{Kg}$ de biomasa seca

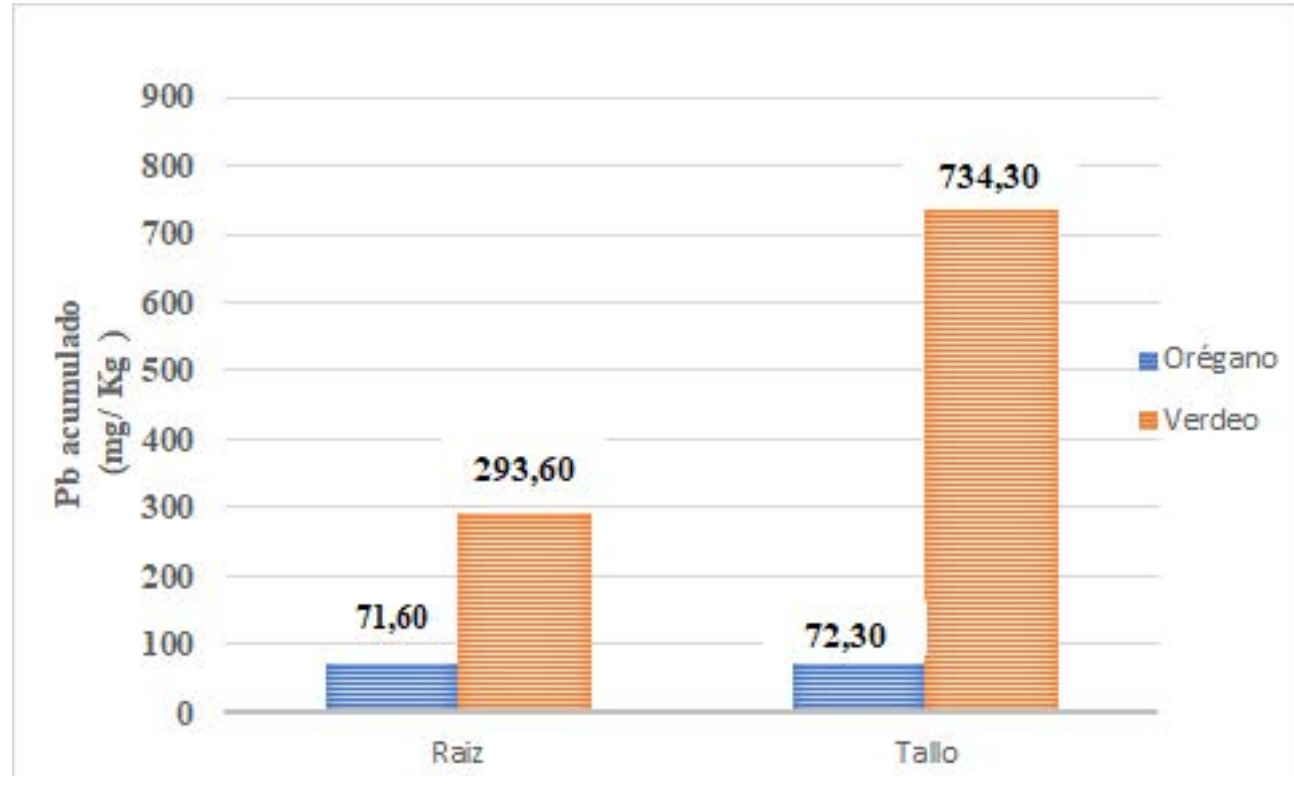

Fuente: Elaboración de la autora

Los valores de acumulación hallados en estos ensayos, si bien representan condiciones ideales, permiten estimar el potencial de cada especie.

De los trabajos requisados, existen reportes de presencia de metales pesados en hortalizas, frutas y otros productos agrícolas que se acercan o superan los valores máximos permitidos para ingesta en los códigos alimentarios de cada país. Por ejemplo, Malik (Malik, 2014) reporta un promedio de acumulación de Plomo en Allium sp. de 0,4 mg/Kg, igual al límite máximo permitido por Estados Unidos. Por su parte, Guerra (FERNANDO GUERRA, 2012) informa un rango de presencia de plomo de 0.5 a $2.5 \mathrm{mg} / \mathrm{Kg}$ en vegetales de la especie Allium en Brasil, que elevan el riesgo toxicológico para la ingesta.

En Argentina, el Codex alimentario (ANMAT, 1953) establece un límite general de $2 \mathrm{mg} / \mathrm{Kg}$ seco de presencia de plomo; mientras que para hortalizas el valor específico permitido es de 0,3 $\mathrm{mg} / \mathrm{Kg}$ seco. Esto implicaría que, en las condiciones ensayadas en este trabajo y teniendo en cuenta el factor de transferencia hallado, el riesgo de intoxicación por ingesta es alto. Nuestros resultados parecerían encontrarse en la misma línea informada por Guerra (FERNANDO GUERRA, 2012), donde el Plomo es uno de los metales con creciente Factor de Transferencia. 
Para los casos donde el suelo presenta baja calidad o está declaradamente contaminado, las opciones en hidroponía suelen ser recomendadas (IZQUIERDO, 2003). Según nuestro estudio, el potencial de acumulación en condiciones de cultivo sin sustrato se acrecienta, con lo que no parecería ser la solución a la obtención de alimentos de mejor calidad, si la calidad de agua utilizada no resguarda parámetros deseables, sobretodo en aquellos sitios donde las instituciones aún no establecieron contacto o donde las huertas establecidas no fueron censadas.

\section{CONSIDERAções finaIS}

De lo expuesto en la sección anterior, puede concluirse que la cebolla de verdeo y el orégano poseen alta capacidad de acumulación de Plomo cuando son cultivados en sustrato $(T F=0,40)$ o en acuaponía.

Estos resultados hacen pensar que es posible diseñar sistemas de fitorremediación basados en estas especies, así como establecer controles mas estrictos de calidad de alimentos.

Los resultados presentados en este trabajo representan los pasos iniciales de una línea de investigación incipiente para Argentina. Los objetivos a corto plazo están centrados en realizar ensayos de mayor duración, bajo condiciones controladas, con Plomo y otros metales a fin de caracterizar la posible acumulación diferencial. A largo plazo, se espera realizar ensayos a campo con las especies trabajadas a fin de diseñar estrategias de manejo de suelos de baja calidad usados en agricultura familiar y estimar el riesgo toxicológico asociado a esta práctica en contextos de contaminación.

\section{REFERENCIAS}

\section{ANMAT. Código Alimentario Argentino 1953.}

DIELEMAN, H. Urban agriculture in Mexico City; balancing between ecological, economic, social and symbolic value. Journal of Cleaner Production, v. 163, p. S156-S163, 2017.

FAGGI, S. L. P. P. M. R. M. C. A. Características del suelo y concentración de metales a lolargo de un gradiente de urbanización en Buenos Aires, Argentina. Multequina, v. 15, p. 69-80, 2006. ISSN 0327-9375.

FAO. Food for the cities- Programa de la FAO para la horticultura urbana y periurbana (HUP). Italia: FAO 2009. 
International Soil Day. 2018. Disponivel em: <http://www.un.org/es/events/soilday/>. Acesso em: 06 mar.

FERNANDO GUERRA, A. R. T., TAKASHI MURAOKA, NERICLENES CHAVES MARCANTE, SOLANGE GUIDOLIN CANNIATTI-BRAZACA. Heavy metals in vegetables and potential risk for human health.

Scientia agricola, v. 69, n. 1, p. 54-60, 2012.

GAISO, F. D. Contaminación por plomo en niños de las villas de la Ciudad Autónoma de Buenos Aires. Auditoría General de la Ciudad de Buenos Aires CABA- Buenos Aires. 2014

GIUFFRÉ, L. M., L.; ROMANIUK, R.; VESPASIANO, C.; SAMMARTINO,F; ARATA, L. Diagnóstico edafológico para la planificación de huertas orgánicas familiares en suelos periurbanos. FAVE- Ciencias agrarias, $v$. 12, n. 1-2, p. 64-76, 2013. ISSN 1666-7719.

GIUFFRÉ, S. R. Y. L. Ambiente y edafología. In: GIUFFRÉ, M. C. Y. L. (Ed.). Edafología: bases y aplicaciones ambientales argentinas. 2da. Argentina: Facultad de Agronomía de la Universidad de Buenos Aires, 2014. cap. 7, ISBN 978-987-29338-9-0.

IGN. Reporte Instituto Geográfico Nacional. 2010

INTA. Plan operativo anual. INTA. Argentina. 2011

Huertas urbanas: producir en casa beneficia a la salud y al ambiente. INTA informa. Argentina: INTA 2018.

IZQUIERDO, C. M. J. La Huerta Hidropónica Popular. Roma, Italia. 2003

JANINE SCHONWALD, F. J. P. Mi casa, mi huerta : técnicas de agricultura urbana NOVELLI, D. Argentina: INTA 2015.

MADALENO, I. Urban agriculture in Belem, Brazil. Cities, v. 17, n. 1, p. 73-77, 2000.

MALIK, A. M. Y. R. N. Human health risk assessment of heavy metals via consumption of contaminated vegetables collected from different irrigation sources in Lahore, Pakistan. Arabian Journal of Chemistry, v. 7, n. ENVIRONMENTAL CHEMISTRY, p. 91-99, 2014.

OWOUR, D. F. Y. S. Farming as a livelihood source for the urban poor of Nakuru, Kenya. Geoforum, v. 39, p. 1978-1990, 2008. 
STORER, D. A simple, high sample volume, ashing, procedure for determining soil organic matter.

Communications on Soil Science Plant Anals, v. 15, p. 759-772, 1984.

XIAOYAN TANG, M. P. L., MURRAY B. MCBRIDE. Arsenic uptake by arugula (Eruca vesicaria, L.) cultivars as affected by phosphate availability. Chemosphere, v. 195, p. 559-566, 2018.

XUEZHU YE, W. X., YONGZHI ZHANG, SHOUPING ZHAO. GANGJUN WANG, QI ZHANG, QIANG WANG. Assessment of heavy metal pollution in vegetables and relationships with soil heavy metal distribution in Zhejiang province, China. Environmetal Monitoring Assessment, v. 187, p. 378, 2014.

YING CHEN, P. W., YUFANG SHAO, YIBIN YING. Health risk assessment of heavy metals in vegetables grown around battery production area. Scientia agricola, v. 71, n. 2, p. 126-132, 2014.

ZELLA, A. Effects of Urban Farming Practices on Income Poverty Reduction in Dodoma Municipality,Tanzania. Current Investigations in Agriculture and Current Research, v. 3, n. 3, p. 354366, 2018. 